\title{
A Spatial Music Listening Experience in Augmented Reality
}

\author{
Lawrence Lim, Wei-Yee Goh, Mara Downing, Misha Sra \\ \{lawrenceklim,weiyee,maradowning,sra\}@ucsb.edu \\ University of California, Santa Barbara
}

\begin{abstract}
Live music provides a more immersive and social experience that recorded music cannot replicate. In a live music setting, listeners perceive sounds differently based on their position with respect to the musicians and can enjoy the experience with others. To make recorded music a dynamic listening experience, we propose and implement an app that adds a spatial dimension to music using augmented reality to allow users to listen to a song as if it were played live. The app lets users place virtual instruments around a physical space and plays the instrument track for each instrument. Users can move around in the space and change the importance of various sound localization aspects to customize their experience. Finally, users can record and livestream to share their listening experience with others.
\end{abstract}

\section{CCS CONCEPTS}

- Human-centered computing $\rightarrow$ Human computer interaction (HCI); Interaction paradigms; Mixed / augmented reality;

\section{KEYWORDS}

Augmented Reality, Audio, Music, Sound Localization

\section{ACM Reference Format:}

Lawrence Lim, Wei-Yee Goh, Mara Downing, Misha Sra. 2021. A Spatial Music Listening Experience in Augmented Reality. In The Adjunct Publication of the 34th Annual ACM Symposium on User Interface Software and Technology (UIST '21 Adjunct), October 10-14, 2021, Virtual Event, USA. ACM, New York, NY, USA, 3 pages. https://doi.org/10.1145/3474349.3480218

\section{INTRODUCTION}

The advent of recorded music has allowed people to enjoy music from the comfort of their homes. However, solitary listening at home does not provide the same level of engagement that live music can due to the lack of an immersive and social environment. In a live music setting, listeners get a spatial experience of sound, hearing the direction and distance of the musicians and their instruments. The music is further augmented by sounds bouncing off venue walls and surfaces. Together, these aspects of locality and acoustics impart listeners a sense of presence and connection during their listening experience.

In this work, we present an augmented reality (AR) application that bridges the gap between recorded and live music by adding a

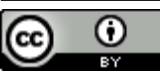

This work is licensed under a Creative Commons Attribution International 4.0 License.

UIST '21 Adjunct, October 10-14, 2021, Virtual Event, USA

(C) 2021 Copyright held by the owner/author(s)

ACM ISBN 978-1-4503-8655-5/21/10.

https://doi.org/10.1145/3474349.3480218 spatial dimension to the at-home listening experience. To provide spatialized audio, we leverage four sound localization techniques: 1) interaural time difference (ITD), 2) interaural level difference (ILD), 3) time differences due to distance, and 4) volume differences due to distance. With a mobile AR device, users can hear the instrument track playing from the virtual instruments which they can freely place in their surroundings. To customize the listening experience, users can modify the sound localization techniques applied to the tracks. Our application also simulates echoes off physical surfaces by using the detected planes in AR. Finally, to explore interactive social features, we tested recording and livestreaming the spatial experience.

Prior work has explored music creation in $\operatorname{AR}[1,4,5]$ and taking piano lessons in AR by superimposing a virtual piano on a real one. ${ }^{1}$ However, our approach differs as we take advantage of sound localization techniques that allow users to perceive sound from the physical location of each virtual instrument. Other works have also implemented and studied sound localization in AR [2, 6]. In contrast, our application offers more customizability for a personalized and social music listening experience.

\section{SYSTEM OVERVIEW}

Our goal is to transform recorded music into a live music listening experience. Our system is composed of the following components: 1) Music source separation, 2) AR application, and 3) Recording and livestreaming. We use the neural network tool Spleeter [3] to split songs into five components (piano, drums, bass, vocals, and other). Each of those components is further split into left and right ear channels using Audacity. ${ }^{2}$ These pre-split audio files are used in the AR application and filtered using sound localization techniques. Rather than using external head-related transfer function (HRTF) tools which provide sound localization, we implemented sound localization in our AR application to allow for user customization. We developed the AR application for iOS 14.6 on Unity 2020.3.3f1 using AR Foundation to place 3D models on detected surfaces. We tested screen recording features using iOS's built-in screen capture features and used Twitch's mobile app to livestream for creating a spatialized and social listening experience.

\subsection{Mobile AR Application}

Our AR application first detects surfaces in the user's physical space, displaying them as blue-bordered planes (Figure 1). Users select instrument icons at the bottom of the screen and tap on a detected plane to place the selected instrument on that surface. The delete button removes a selected instrument from the AR scene.

\footnotetext{
${ }^{1}$ Massive Technologies Inc. 2021. AR Pianist - 3D Piano Concerts [Mobile app]. (Version 2.0.8). Retrieved from https://apps.apple.com/us/app/ar-pianist-3d-piano-concerts/ id1478118473.

${ }^{2}$ Audacity Team. 2021. Audacity(R): Free Audio Editor and Recorder [Computer application]. (Version 3.0.3). Retrieved from https://www.audacityteam.org.
} 


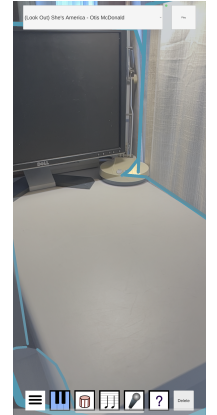

(a)

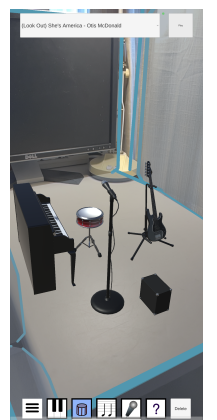

(b)

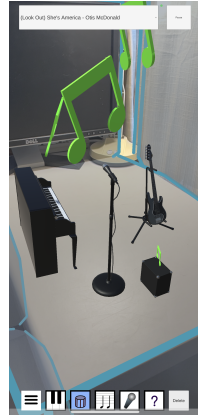

(c)

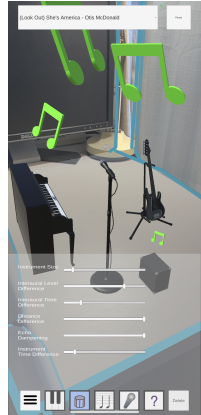

(d)
Figure 1: (a) Empty AR scene. (b) Instruments placed on physical surfaces. (c) Instruments playing spatialized music visually represented by animated music notes. (d) Advanced app settings enable users to customize the model size, degree of sound localization, and volume of echoes.

Once the instruments are placed in the physical environment, users can select among the pre-split songs using the dropdown menu and play them using the play and pause toggle at the top right. An animated green music note hovers over the instruments to indicate that music is playing. As users move around the scene, the tracks audibly change based on the phone's position with respect to the instruments due to the sound localization techniques. Users can customize sound localization and instrument sizes using sliders found in the advanced settings menu, which can be opened with the bottom left hamburger button (Figure 1d). Adjusting the model size allows users to either arrange miniature instrument models on desks or physically traverse through life-sized instruments that can be placed in larger indoors and outdoors environments.

\section{IMPLEMENTATION}

Our application uses recorded music to create a novel spatialized listening experience closer to live music. We spatially attached instrument tracks to virtual instruments and implemented sound localization techniques to provide a sense of locality similar to a live performance. To simulate realistic acoustics, we also implemented echoes from detected planar surfaces in the real world environment.

\subsection{Sound Localization}

Two known techniques we implemented for sound localization are interaural time difference (ITD) and interaural level difference (ILD). ITD delays the sound that reaches a user's further ear to give the perception that the sound had to travel farther to reach that ear. ILD dampens the volume in the further ear in contrast to the closer ear. We also implemented sound localization involving both volume and time differences based on distance: Farther sound sources arrive in our ears both later in time and with less intensity. To give users the ability to control the level of sound localization, we implemented sliders to adjust the contribution of each technique. As users tilt their phone in different directions, they can discern direction of the sound with ILD and ITD as if they were turning their head. When users move their phone closer to a virtual instrument, they hear

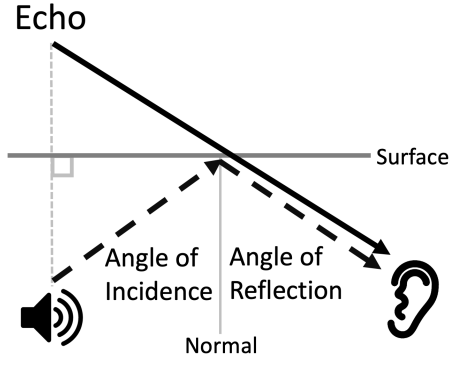

louder music as if they were walking towards a musician at a live performance venue.

\subsection{Echoes}

Our application simulates echoes by bouncing sound from detected flat surfaces in the user's environment. We simulate each echo by placing another audio source behind each surface in the direction of the echo. This results in an echo similar to light reflecting off mirrors where the angle of incidence is equal to the angle of reflection (Figure 2). The same sound localization techniques described previously apply to echoes as well, causing users to hear sound bounce off multiple surfaces delayed, attenuated, and from their respective direction. This imparts users at home with acoustics similar to a music venue.

\section{RECORDING AND LIVESTREAMING}

In order to test social features that allow music sharing, we investigated external applications such as iOS's screen capture and Twitch's mobile app. Crucially, these tools were able to maintain the spatial audio output of our application whereas other tools converted the stereo audio to mono audio in their pipeline. In our testing, the applications also retain the video and audio quality so that people can comfortably interact during the shared music listening experience.

\section{CONCLUSION AND FUTURE WORK}

In this work we presented an $\mathrm{AR}$ application that transforms recorded music to a spatial listening experience akin to a live music setting. We achieved this by implementing sound localization, simulating echoes, and testing social features for our application. Although our implementation of spatialized audio is not as optimized as external HRTF libraries, further testing and enhancements can lead to greater improvements in this area. We also plan to integrate music source separation into our pipeline to allow users to import their own song libraries for a spatial listening experience. Since our application only supports five categories of instruments presently, we plan to use improved music source separation models trained on datasets with more categories so that we can support a wider range of instruments. Finally, integrating social features into our application pipeline as well as extending our work into spatial music composition will enable more creative interactions such as live personalized performances that anyone can create in their own home environments. 


\section{REFERENCES}

[1] Robert Clouth. 2013. Mobile Augmented Reality as a Control Mode for Real-time Music Systems. Master's thesis. Universitat Pompeu Fabra, Barcelona.

[2] Daniel Dobler, Michael Haller, and Philipp Stampfl. 2002. ASR: augmented sound reality. In ACM SIGGRAPH 2002 conference abstracts and applications. 148-148.

[3] Romain Hennequin, Anis Khlif, Felix Voituret, and Manuel Moussallam. 2020. Spleeter: a fast and efficient music source separation tool with pre-trained models. fournal of Open Source Software 5, 50 (2020), 2154
[4] Tomás Laurenzo, Ernesto Rodríguez, and Juan Fabrizio Castro. 2009. YARMI: an Augmented Reality Musical Instrument. In Proceedings of the International Conference on New Interfaces for Musical Expression. 268-269.

[5] Sihwa Park. 2019. ARLooper: a mobile AR application for collaborative sound recording and performance. In Proceedings of the International Symposium on Computer Music Multidisciplinary Research. 233-240.

[6] Yolanda Vazquez-Alvarez, Ian Oakley, and Stephen A Brewster. 2012. Auditory display design for exploration in mobile audio-augmented reality. Personal and Ubiquitous computing 16, 8 (2012), 987-999. 